\title{
Effects of Randomness on the Field-Induced Phase Transition in the $S=1$ Bond-Alternating Spin Chain NTENP
}

\author{
Shinichi Matsubara ${ }^{1}$, Katsuaki Kodama ${ }^{1 *}$, Masashi Takigawa ${ }^{1 \dagger}$, and Masayuki Hagrwara ${ }^{2}$ \\ ${ }^{1}$ Institute for Solid State Physics, University of Tokyo, Kashiwa, Chiba 277-8581, Japan \\ ${ }^{2}$ KYOKUGEN, Osaka University, Toyonaka, Osaka 560-8531, Japan
}

\begin{abstract}
We report novel effects of randomness in the $\mathrm{S}=1$ bond-alternating antiferromagnetic chain compound with a dimer-singlet ground state [Ni(N,N'-bis(3-aminopropyl)propane-1,3diamine $\left.\left(\mu-\mathrm{NO}_{2}\right)\right] \mathrm{ClO}_{4}$ abbreviated as NTENP. The ${ }^{15} \mathrm{~N}$ NMR spectra develop a continuum with sharply peaked edges at low temperatures, indicating an inhomogeneous staggered magnetization induced by magnetic field. We attribute this to random anisotropic interactions due to disorder of $\mathrm{NO}_{2}$ groups in the chains. The field-induced antiferromagnetic transition exhibits remarkably anisotropic behavior. We propose that a field-induced incoherent magnetization is transformed into a coherent antiferromagnetic moment with spatially fluctuating amplitude.
\end{abstract}

KEYWORDS: NTENP, random effects, field-induced magnetic transition, nuclear magnetic resonance

Among various quasi-one-dimensional quantum antiferromagnets, $\mathrm{S}=1$ bond-alternating chains expressed by the Hamiltonian

$$
\mathcal{H}_{0}=J \sum_{i}\left(\boldsymbol{S}_{2 i-1} \cdot \boldsymbol{S}_{2 i}+\alpha \boldsymbol{S}_{2 i} \cdot \boldsymbol{S}_{2 i+1}\right)
$$

has an interesting feature that two distinct singlet phases appear as the bond alternating ratio $\alpha$ varies from zero to one. ${ }^{1}$ The dimer-singlet phase is realized for $\alpha<$ $\alpha_{c}=0.588,{ }^{2}$ while the Haldane phase with the valencebond-solid states appears for $\alpha>\alpha_{c}$. The triplet excitations have a finite energy gap except at $\alpha=\alpha_{c}{ }^{3}$

The triplet excitations are split by a magnetic field $(\boldsymbol{H})$. The energy of the lowest branch is reduced with the field and vanishes at a critical field $H_{c}$. For a finite interchain coupling, the Bose-Einstein condensation (BEC) of the triplets occurs generally for $H>H_{c} \cdot{ }^{4,5}$ Since the bosons are created by the transverse spin operator at the antiferromagnetic wave vector, the BEC is equivalent to an antiferromagnetic (AF) order perpendicular to the magnetic field. While this is a generic scenario, the field-induced AF transitions in real materials show a variety of behavior depending on various perturbations such as anisotropy, frustrating interactions, and disorder, which are not included in eq. (1).

The single ion anisotropy energy in a uniaxial $\mathrm{S}=1$ system is given by $D \sum_{i} S_{i z}^{2}$. For $H \| z$ the AF order perpendicular to the field has the $X Y$-symmetry, while for $H \perp$ $z$ the direction of the AF moment is uniquely fixed, resulting in the Ising symmetry. Other types of anisotropy are allowed for crystals with low symmetry, for example, the Zeeman interaction involving anisotropic $g$-tensors $\boldsymbol{H} \cdot \sum_{i} \boldsymbol{g}_{i} \cdot \boldsymbol{S}_{i}$ and the Dzyaloshinskii-Moriya (DM) interaction $^{6,7} \sum_{i} \boldsymbol{d}_{i} \cdot\left(\boldsymbol{S}_{i} \times \boldsymbol{S}_{i+1}\right)$. If such interactions contain a staggered part with sign alternation, $\boldsymbol{g}_{i}=\boldsymbol{g}^{u}+(-1)^{i} \boldsymbol{g}^{s}$ or $\boldsymbol{d}_{i}=\boldsymbol{d}^{u}+(-1)^{i} \boldsymbol{d}^{s}$, application of a uniform magnetic field leads to an effective staggered field $(-1)^{i} \boldsymbol{H}_{s}$ with $\boldsymbol{H}_{s} \propto \boldsymbol{g}^{s} \cdot \boldsymbol{H}$ or $\boldsymbol{H}_{s} \propto \boldsymbol{d}_{s} \times \boldsymbol{H} / J .^{8}$ The staggered field

*Present address: Neutron Science Research Center, JAERI.

${ }^{\dagger}$ E-mail address: masashi@issp.u-tokyo.ac.jp produces a staggered magnetization even for $H<H_{c}$. It also mixes the singlet with the triplets, preventing the gap from closing at $H_{c} .{ }^{9,10}$ These phenomena have been observed, for example, in the Haldane chain compound $\mathrm{Ni}\left(\mathrm{C}_{2} \mathrm{H}_{8} \mathrm{~N}_{2}\right)_{2} \mathrm{NO}_{2}\left(\mathrm{ClO}_{4}\right)$ (NENP). ${ }^{9,} 10$ (Similar phenomena have been observed also in the $2 \mathrm{D}$ frustrated dimer spin system $\left.\mathrm{SrCu}_{2}\left(\mathrm{BO}_{3}\right)_{2} \cdot{ }^{11,12}\right)$ Such diversity of the quantum critical phenomena in real materials is a fascinating aspect of the physics of spin systems in magnetic fields.

[Ni(N,N'-bis(3-aminopropyl)propane-1,3-diamine ( $\mu$ $\left.\left.\mathrm{NO}_{2}\right)\right] \mathrm{ClO}_{2}$ abbreviated as NTENP with the chemical formula $\mathrm{Ni}\left(\mathrm{C}_{9} \mathrm{H}_{24} \mathrm{~N}_{4}\right)\left(\mathrm{NO}_{2}\right)\left(\mathrm{ClO}_{4}\right)$ is an example of $\mathrm{S}=1$ bond-alternating chain systems. The chains, separated by $\mathrm{ClO}_{4}^{-}$anions, run along the $a$-axis of the triclinic $P \overline{1}$ structure, where the $\mathrm{Ni}^{2+}$ ions are connected via nitrito groups $\left(\mathrm{NO}_{2}^{-}\right)$with alternation of short and long bonds (Fig. 1). ${ }^{13}$ The results of susceptibility, magnetization, ${ }^{14}$ and neutron inelastic scattering ${ }^{15}$ for NTENP, as well as the ESR data for Zn-doped NTENP ${ }^{14}$ established that NTENP belongs to the dimer-singlet phase with the exchange parameters $\alpha=0.45, J=54 \mathrm{~K}$ and the nearly uniaxial single-ion anisotropy $D=13.6 \mathrm{~K}$. The latter

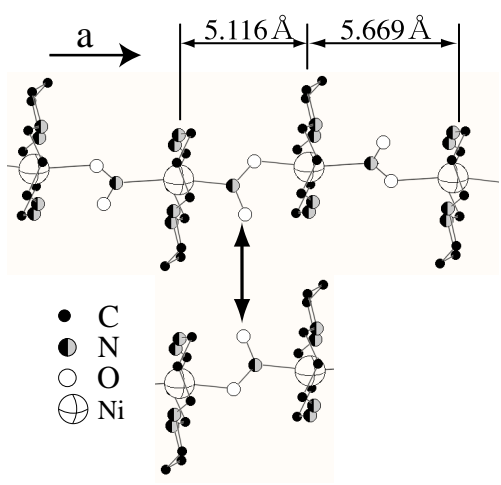

Fig. 1. The structure of a magnetic chain in NTENP. Two types of $\mathrm{Ni}-\mathrm{NO}_{2}-\mathrm{Ni}$ bonds related by inversion occur randomly. Note that individual bonds do not have the inversion symmetry. 

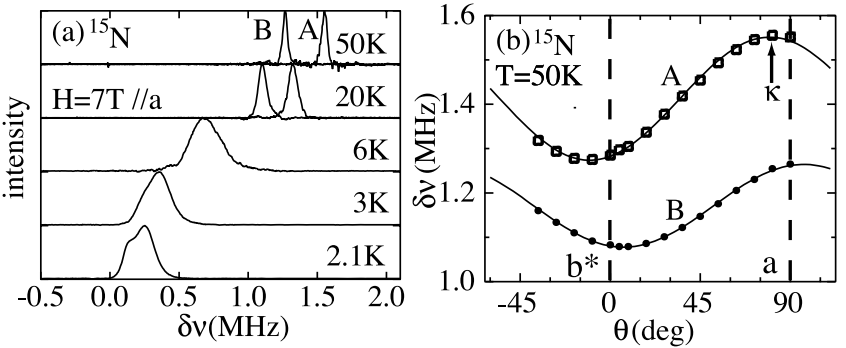

Fig. 2. (a) The ${ }^{15} \mathrm{~N}$ NMR spectra at $\mathrm{H}=7 \mathrm{~T}$ along the $a$-axis at several temperatures. (b) Angular dependence of $\delta \nu$ at $T=50 \mathrm{~K}$ for the field in the $a b^{*}$-plane. A and $\mathrm{B}$ denotes the different sites. The lines show sinusoidal curves with the period of $180^{\circ}$.

is consistent with the critical fields for the onset of magnetization, $H_{c}=9.3 \mathrm{~T}$ for $\boldsymbol{H} \| \boldsymbol{a}$ and $H_{c}=12.4 \mathrm{~T}$ for $\boldsymbol{H} \perp \boldsymbol{a},{ }^{14}$ and the triplly split excitation gap energies, $1.06,1.15$, and $1.96 \mathrm{meV}$, observed by the neutron scattering experiments. ${ }^{16}$ The specific heat shows a pronounced sharp peak at low temperatures for $H>H_{c},{ }^{17}$ indicating a well developed long range $\mathrm{AF}$ order. The $\mathrm{AF}$ order, which was confirmed by neutron diffraction, ${ }^{16}$ can be interpreted as the BEC of triplets. NTENP also has a unique structural feature. ${ }^{13}$ A nitrogen atom on each bond can be located next to either the left or the right $\mathrm{Ni}$ atom randomly with equal probability (Fig. 1). However, no effect of randomness has been reported so far.

In this paper, we report observation of a field-induced inhomogeneous staggered magnetization in a single crystal of NTENP by nuclear magnetic resonance (NMR) experiments on ${ }^{15} \mathrm{~N}$ (nuclear spin $\left.I=1 / 2\right)$ and ${ }^{35} \mathrm{Cl}(I=3 / 2)$ nuclei. We attribute this to the structural randomness, which also causes highly anisotropic behavior of the fieldinduced magnetic transition. Only the $\mathrm{N}$ atoms in the $\mathrm{NO}_{2}^{-}$groups were replaced by ${ }^{15} \mathrm{~N}$ isotope. The NMR spectra were obtained from the Fourier transform of the spin-echo signal. The nuclear relaxation rate $1 / T_{1}$ was measured by the inversion recovery method.

The ${ }^{15} \mathrm{~N}-\mathrm{NMR}$ spectra are shown in Fig. 2(a) at various temperatures for the field of $7 \mathrm{~T}$ along the $a$-axis. Here the signal intensity is plotted against the frequency shift $\delta \nu=\nu-\gamma_{N} H$, where $\nu$ is the signal frequency and $\gamma_{N}$ is the nuclear gyromagnetic ratio $(4.3142 \mathrm{MHz} / \mathrm{T}$ for ${ }^{15} \mathrm{~N}$ and $4.1717 \mathrm{MHz} / \mathrm{T}$ for ${ }^{35} \mathrm{Cl}$ ). The signal frequency of a nucleus is proportional to the sum of the external field and the time-averaged magnetic hyperfine field due to Ni spins, $\nu=\gamma_{N}\left(\left|\boldsymbol{H}+\left\langle\boldsymbol{H}^{h f}\right\rangle\right|\right)$ with $\left\langle\boldsymbol{H}^{h f}\right\rangle=\sum_{i} \boldsymbol{A}_{i} \cdot \boldsymbol{g} \cdot\left\langle\boldsymbol{S}_{i}\right\rangle$, where $\boldsymbol{A}_{i}$ is the hyperfine coupling tensor between the nucleus and the nearby spin $\boldsymbol{S}_{i}$ and \langle\rangle stands for the time-average. Since $H \gg\left|\left\langle\boldsymbol{H}^{h f}\right\rangle\right|$, the frequency shift is given by the projection of $\left\langle\boldsymbol{H}^{h f}\right\rangle$ along the external field direction, $\delta \nu=\boldsymbol{H} \cdot\left\langle\boldsymbol{H}^{h f}\right\rangle / H$. The NMR spectra then represent the distribution of $\delta \nu$.

Two NMR lines are observed at $50 \mathrm{~K}$, corresponding to the distinct $\mathrm{N}$ sites on the short and the long bonds with different hyperfine coupling tensors to the uniform magnetization $\boldsymbol{A}_{u}=\sum_{i} \boldsymbol{A}_{i}$. The A-site with the larger shift is likely to be on the short bond. The shifts at both sites are reduced to nearly zero at low temperatures, where two lines overlap (Fig. 2(a)), in a way similar to the susceptibility. However, the line width increases with decreasing temperature, indicating inhomogeneous distribution of magnetization at low temperatures. We discuss the line width in more detail below.

In Fig. 2(b), $\delta \nu$ at $T=50 \mathrm{~K}$ is plotted as a function of the angle $\theta$ between the $b^{*}$-axis and the field in the $a b^{*}$-plane. For the A-site, $\delta \nu$ has a maximum at $\theta=80^{\circ}$ (denoted as $\kappa$ ). The global maximum in the plane containing the $\kappa$-axis and perpendicular to the $a b^{*}$-plane occurs at $4^{\circ}$ from the $\kappa$-axis (defined as $\xi$, not shown), which should then be one of the principal axes of $\boldsymbol{A}_{u}$. The standard $K-\chi$ analysis yields the principal value as $A_{u}^{\xi \xi}=4.2 \mathrm{~T} / \mu_{B}$. Other principal values are obtained as 2.9 and $3.1 \mathrm{~T} / \mu_{B}$. Thus $\boldsymbol{A}_{u}$ is approximately uniaxial along the Ni-N bond direction with a large isotropic part. Since these values are an order of magnitude larger than the dipolar field, the major source of the hyperfine field should be the spin density on the $2 \mathrm{~s}$ and the $2 \mathrm{p}$ states of $\mathrm{N}$ sites transferred from the $\mathrm{d}$ states of the neighboring $\mathrm{Ni}$ sites. We expect the same mechanism for the B-sites based on the similar magnitude and $\theta$-dependence of $\delta \nu$ in the $a b^{*}$-plane, although the complete angular dependence has not been measured.

To understand the mechanism for the line broadening at low temperatures, we examined angular dependence of the line shape at $1.6 \mathrm{~K}$ for the field in the $a b^{*}$-plane as shown in Fig. 3(a). When the field is away from the $a$ or $b^{*}$-axes, the spectra develop a double peak structure with a continuum and sharp edges on both sides. The largest peak splitting at $\theta=45^{\circ}(\sim 2 \mathrm{MHz})$ is about two orders of magnitude larger than the width of individual lines at $50 \mathrm{~K}$, while the bulk magnetization at $1.6 \mathrm{~K}$ is an order of magnitude smaller than the value at $50 \mathrm{~K}$. Such broadening must be due to continuous distribution of the hyperfine field with a large weight near the extremal values. The nearly symmetric line shape with respect to $\delta \nu=0$ indicates that its origin is a local staggered magnetization with spatial inhomogeneity. We observed that the line width changes linearly with the field, therefore, the staggered magnetization must be induced by the external field.

The angular dependence of the line shape strongly suggests that the local staggered magnetization is perpendicular to the field. If it were parallel to the field, the peak splitting should be largely independent of the field direction since the hyperfine coupling tensor is dominantly isotropic. On the other hand, splitting due to perpendicular magnetization is caused only by the anisotropic part of the hyperfine coupling. The more the field deviates from the principal axes of the hyperfine coupling tensor, the larger should become the splitting. This is precisely what we observed. Upon closer inspection, however, we note that the spectrum becomes narrowest for $\boldsymbol{H} \| \boldsymbol{a}$, even though the $a$-axis deviates slightly from the principal axes. In contrast, clear splitting is observed for $\boldsymbol{H} \| \boldsymbol{b}^{*}$ while the $b^{*}$-axis deviates from the closest principal axis by a similar amount as the $a$-axis. This suggests that the magnitude of the staggered magnetization varies with the field direction and becomes smallest for $\boldsymbol{H} \| \boldsymbol{a}$.

To confirm this point, we examined the ${ }^{35} \mathrm{Cl}$ NMR 

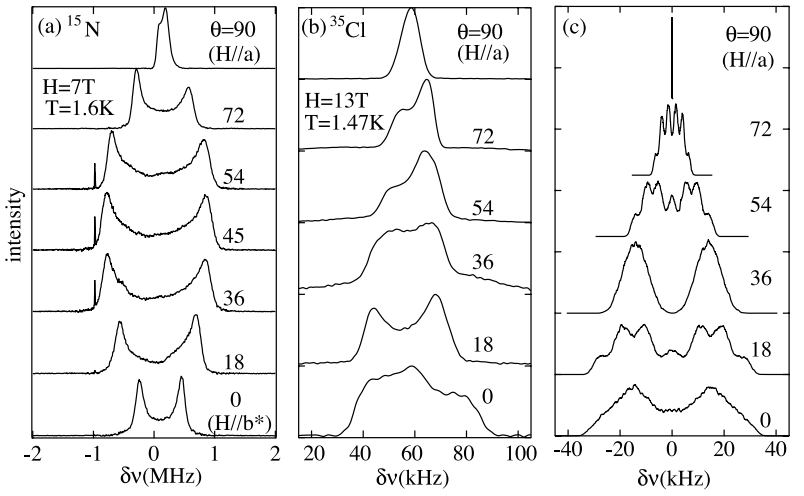

Fig. 3. Angular dependence of the NMR spectra for the field in the $a b^{*}$-plane. (a) ${ }^{15} \mathrm{~N}$ spectra at $T=1.6 \mathrm{~K}$ and $H=7 \mathrm{~T}$. The sharp peak near $-1 \mathrm{MHz}$ is due to ${ }^{35} \mathrm{Cl}$ nuclei. (b) ${ }^{35} \mathrm{Cl}$ spectra at $T=1.4 \mathrm{~K}$ and $H=13 \mathrm{~T}$. (c) The simulated ${ }^{35} \mathrm{Cl}$ spectra for the model described in the text.

spectra. Since a $\mathrm{Cl}$ nucleus is coupled to many $\mathrm{Ni}$ spins via dipolar interaction, the hyperfine field should be less sensitive to the direction of the random magnetization. The angular variation of the ${ }^{35} \mathrm{Cl} \mathrm{NMR}$ spectra of the central transition $\left(I_{z}=-1 / 2 \leftrightarrow 1 / 2\right)$ at $T=1.47 \mathrm{~K}$ are shown in Fig. 3(b). Although the field (13 T) exceed $H_{c}$, no long range AF order occurs at this temperature. ${ }^{17}$ The line width shows a minimum for $\boldsymbol{H} \| \boldsymbol{a}$ and a maximum for $\boldsymbol{H} \| \boldsymbol{b}^{*}$ in contrast to the case of ${ }^{15} \mathrm{~N}$ spectra. This supports that the staggered magnetization is minimized for $\boldsymbol{H} \| \boldsymbol{a}$.

The field-induced inhomogeneous staggered magnetization observed by NMR can be qualitatively understood by the structural disorder mentioned before. Since the individual Ni-Ni bond breaks the inversion symmetry, the following DM interaction should be allowed

$$
\mathcal{H}_{D M}=\sum_{j}\left[ \pm \boldsymbol{d}_{1} \cdot\left(\boldsymbol{S}_{2 i-1} \times \boldsymbol{S}_{2 i}\right) \pm \boldsymbol{d}_{2} \cdot\left(\boldsymbol{S}_{2 i} \times \boldsymbol{S}_{2 i+1}\right)\right],
$$

where the first (second) term applies to the short (long) bond and the random sign arises from the two configurations of $\mathrm{NO}_{2}$ groups related by inversion. The structural disorder should cause random modulation of the $g$-tensor as well. Although this will also lead to a random staggered magnetization, how the $g$-tensor depends on bond configurations is not obvious and we leave the detailed analysis for future studies.

We first consider the simplest case of isolated dimers $(\alpha=0)$ with $\boldsymbol{d}_{1} \| \boldsymbol{a}$. The external field induces staggered magnetization with the random $\operatorname{sign}^{8,11,12}\left\langle\boldsymbol{S}_{2 i-1}\right\rangle-$ $\left\langle\boldsymbol{S}_{2 i}\right\rangle= \pm\left(8 g / 3 J^{2}\right) \boldsymbol{d}_{1} \times \boldsymbol{H}= \pm M_{s} \cos \theta$. We have simulated the ${ }^{35} \mathrm{Cl}$ spectra due to the dipolar field from such magnetization with $M_{s}=0.4 \mu_{B}$ as shown in Fig. 3(c). Even this simplest model reproduces qualitative feature of the data, providing estimation for the size of the staggered magnetization. However, this does not account for the spectra of ${ }^{15} \mathrm{~N}$ nuclei, which should couple dominantly to two neighboring $\mathrm{Ni}$ spins. For the B-sites on the weak bonds, the sign change of the staggered magnetization leads to a line splitting symmetric about $\delta \nu=0$. Contrary, the hyperfine field at the A-sites on the strong

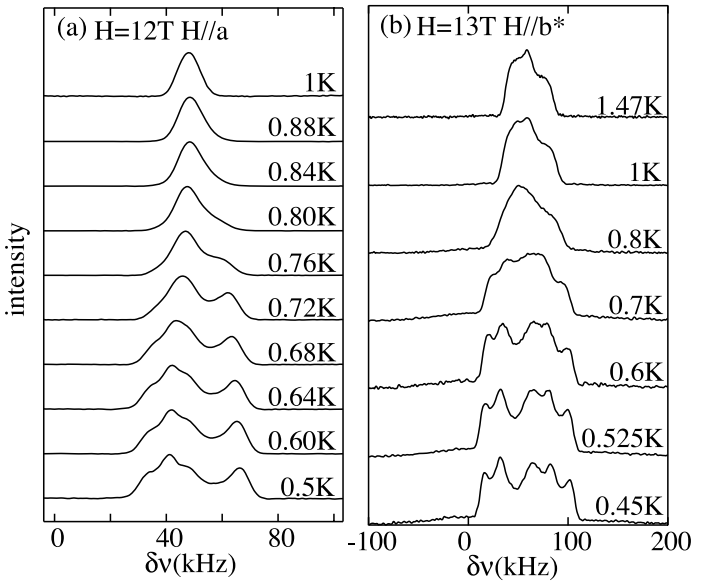

Fig. 4. Temperature variation of the ${ }^{35} \mathrm{Cl}$ NMR spectra across the of phase transition for (a) $H=12 \mathrm{~T}$ along the $a$-axis and (b) $H=13 \mathrm{~T}$ along the $b^{*}$-axis.

bonds should not be altered by the inversion that flips the staggered magnetization of the same bond. We then expect a superposition of doubly peaked and singly peaked spectra, inconsistent with the data.

However, the interdimer interaction is not weak in NTENP $(\alpha \sim 0.5)$ and one AF correlation length should contain several dimers. The random perturbation (eq. 2) may enhance the AF correlation set by the main Hamiltonian (eq. 1) in some regions, but may frustrate with it in other regions. This competition (frustration) will bring spatial fluctuations both in the correlation length and in the size of the staggered magnetization. Such distribution is likely to produce continuous spectra for both $\mathrm{N}$ sites, although more precise understanding of the ${ }^{15} \mathrm{~N}$ spectra based on a realistic model is highly desired. We emphasize that long range coherence should never be established for the field-induced magnetization below $H_{c}$, therefore, it cannot be detected by neutron diffraction.

We found that such incoherent staggered magnetization has profound effects on the field-induced AF transition. Evolution of the ${ }^{35} \mathrm{Cl} \mathrm{NMR}$ spectra upon entering into the $\mathrm{AF}$ phase by lowering temperature is shown in Fig. 4 for (a) $H=12 \mathrm{~T}$ along the $a$-axis and (b) $H=13 \mathrm{~T}$ along the $b^{*}$-axis. The transition temperature $T_{c}$ can be inferred from the specific heat data ${ }^{17}$ to be $0.86 \mathrm{~K}$ (case a) and $0.88 \mathrm{~K}$ (case b). For the case (a), the spectrum gets broadened significantly in a relatively narrow temperature range below $\mathrm{T}_{c}(0.84 \mathrm{~K} \geq T \geq 0.72 \mathrm{~K})$ and broad double peak structure appears at lower temperatures. If there were no disorder, an $\mathrm{AF}$ phase transition generally causes NMR lines to split and the splitting is proportional to the $\mathrm{AF}$ order parameter. In the presence of substantial disorder, an AF order results in line broadening rather than splitting. Thus the data for the case (a) are consistent with what one would expect for a second order AF transition with strong disorder. For the case (b), however, the spectrum has a broad shape already above $T_{c}$ (Fig. $3 \mathrm{~b}$ ). Only a minor change occurs in a wide temperature range below $T_{c}$. One would not have noticed a phase transition from the NMR spectra alone.

The peculiar behavior for $\boldsymbol{H} \| \boldsymbol{b}^{*}$ can be qualitatively 


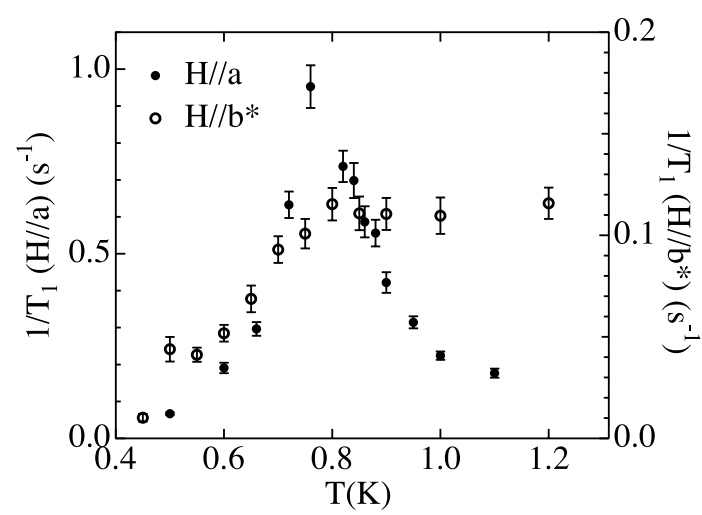

Fig. 5. The temperature dependence of $1 / T_{1}$ for ${ }^{35} \mathrm{Cl}$ nuclei for $H=12 \mathrm{~T}$ parallel to the $a$-axis (solid circles, left scale) and $H=13 \mathrm{~T}$ parallel to the $b^{*}$-axis (open circles, right scale).

understood if one realizes that an incoherent staggered magnetization is already developed above $T_{c}$. Below $T_{c}$, the long range coherence must be established as indicated by the sharp Bragg peaks of neutron diffraction. ${ }^{16}$ However, there should still be large spatial fluctuations in the magnitude due to frustration between the regular $\mathrm{AF}$ interactions and the random anisotropic interactions. Since the NMR spectrum is sensitive only to local magnetization profile, the states above and below $T_{c}$ may not be clearly distinguishable by NMR . More normal behavior is expected for $\boldsymbol{H} \| \boldsymbol{a}$ because the staggered magnetization above $T_{c}$ is minimum. Usually the alternating DM interaction without disorder inhibits a phase transition as has been observed in NENP. It is interesting that in NTENP the disorder enables the phase transition.

The field-induced AF transition exhibits remarkable anisotropy also in the dynamical properties. Temperature dependence of the nuclear spin-lattice relaxation rate $1 / T_{1}$ for ${ }^{35} \mathrm{Cl}$ nuclei is shown in Fig. 5 . We found that the nuclear magnetization follows an exponential time dependence after it recovers to about $50 \%$ of the equilibrium value, even though the initial recovery was non-exponential. For $\boldsymbol{H} \| \boldsymbol{a}, 1 / T_{1}$ shows a huge enhancement near $T_{c}$ due to slowing down of spin fluctuations. It seems unlikely that the enhancement of $1 / T_{1}$ over such a wide temperature range is related to $3 \mathrm{D}$ ordering. We speculate that it may be due to enhanced $1 \mathrm{D}$ fluctuations in the Luttinger liquid regime, since the field direction maintains the approximate XY-symmetry.

In contrast, no enhancement was observed for $\boldsymbol{H} \| \boldsymbol{b}^{*}$. $1 / T_{1}$ stays nearly constant down to $T_{c}$, below which it is depressed rapidly. This behavior could arise from two reasons. First, the field-induced AF order has the Ising symmetry for $\boldsymbol{H} \| \boldsymbol{b}^{*}$, leading to a narrower critical temperature range. However, this alone would not eliminate the enhancement of $1 / T_{1}$ completely. Second, the spin fluctuations above $T_{c}$ should be suppressed significantly by the field-induced random magnetization. We suppose that the incoherent magnetization above $T_{c}$ is transformed into a coherent $\mathrm{AF}$ moment below $T_{c}$ by the reversal of magnetization of domains whose size is set by the locally varying correlation length. The constant behavior of $1 / T_{1}$ above $T_{c}$ indicates that this process does not involve slowing down of fluctuations. Nevertheless, the phase transition can be identified clearly by the sudden drop of $1 / T_{1}$ below $0.8 \mathrm{~K}$. We notice that the temperature for the anomaly of $1 / T_{1}(0.76 \mathrm{~K}$ for $\boldsymbol{H} \| \boldsymbol{a}$ and $0.8 \mathrm{~K}$ for $\boldsymbol{H} \| \boldsymbol{b}^{*}$ ) is slightly lower than $T_{c}$ determined by specific heat. We do not understand the reason at moment. Detailed NMR measurements of the phase diagram and comparison with other experimental results remain to be done in future.

In conclusion, anomalous line broadening of the ${ }^{15} \mathrm{~N}$ and ${ }^{35} \mathrm{Cl}$ NMR spectra in NTENP provide evidence for field-induced incoherent staggered magnetization due to structural disorder. We propose that the anisotropic response of this random magnetization significantly affects both the static and the dynamic behavior of the field-induced antiferromagnetic transition. The phenomena found in NTENP resemble the "random field effects" studied extensively a few decades ago ${ }^{18}$ in that both are caused by frustration between the regular AF interactions and the random magnetic field. In NTENP, however, the random interaction appears to have no effects on the singlet ground state at zero-field. The magnetic field induces both the AF order and the effective random field. We believe that this is a new type of quantum phase transition involving randomness and calls for serious theoretical investigations. We would like to thank J. Yamaura for help in crystal orientation by X-ray measurements.

1) I. Affleck and F. D. M. Haldane: Phys. Rev. B 36 (1987) 5291.

2) M. Kohno, M. Takahashi, and M. Hagiwara; Phys. Rev. B57 (1998) 1046.

3) M. Hagiwara, Y. Narumi, K. Kindo, M. Kohno, H. Nakano, R. Sato, and M. Takahashi; Phys. Rev. Lett. 80 (1998) 1312.

4) I. Affleck: Phys. Rev. B 43 (1991) 3215.

5) T. Nikuni, M. Oshikawa, A. Oosawa, and H. Tanaka: Phys. Rev. Lett. 84 (2000) 5368.

6) I. Dzyaloshinskii: J. Phys. Chem. Solid 4 (1958) 241.

7) T. Moriya: Phys. Rev. 120 (1960) 91.

8) H. Huang and I. Affleck: Phys. Rev. B 69 (2004) 184414.

9) M. Chiba, Y. Ajiro, H. Kikuchi, T. Kubo, and T. Morimoto: Phys. Rev. B 44 (1991) 2838.

10) P. P. Mitra and B. I. Halperin: Phys. Rev. Lett. 72 (1994) 912.

11) S. Miyahara, F. Mila, K. Kodama, M. Takigawa, M. Horvatić, C. Berthier, H. Kageyama, and Y. Ueda: J. Phys.:Condens. Matter 16 (2004) S911.

12) K. Kodama, S. Miyahara, M. Takigawa, M. Horvatić, C. Berthier, F. Mila, H. Kageyama, and Y. Ueda: J. Phys.: Condens. Matter 17 (2005) L61.

13) A. Escuer, R. Vicente, and X. Solans: J. Chem. Soc. Dalton Trans., 1997531.

14) Y. Narumi, M. Hagiwara, M. Kohno, and K. Kindo: Phys. Rev. Lett. 86 (2001) 324.

15) A. Zheludev, T. Masuda, B. Sales, D. Mandrus, T. Papenbrock, T. Barnes, and S. Park: Phys. Rev. B 69 (2004) 144417.

16) M. Hagiwara, L. R. Regnault, A. Zheludev, A. Stunault, N. Metoki, T. Suzuki, S. Suga, K. Kakurai, Y. Koike, P. Vorderwisch, and J. H. Chung: Phys. Rev. Lett. 94 (2005) 177202.

17) N. Tateiwa, M. Hagiwara, H. Aruga-Katori, and T. C. Kobayashi: Physica B 329-333 (2003) 1209.

18) A. Aharony: J. Mag. Mag. Mat. 54-57 (1986) 27. 